\title{
Tratamento cirúrgico de queratocisto odontogênico associado a crioterapia com gás refrigerante: acompanhamento de 10 anos
}

Surgical treatment of odontogenic keratocyst associated with cryotherapy with refrigerant gas: 10year follow-up

Tratamiento quirúrgico del queratoquisto odontogenico asociado a crioterapia con gas refrigerante: seguimiento a 10 años

Jose Carlos Garcia de Mendonça

ORCID: https://orcid.org/0000-0003-3217-872X Universidade Federal do Mato Grosso do Sul, Brasil E-mail: jose.mendonca@ufms.br

Ellen Cristina Gaetti Jardim

ORCID: https://orcid.org/0000-0003-2471-465X Universidade Federal do Mato Grosso do Sul, Brasil E-mail: ellen.jardim@ufms.br

Julio Cesar Leite da Silva

ORCID: https://orcid.org/0000-0002-9835-7432 Universidade Federal de Mato Grosso do Sul, Brasil E-mail: julio.silva@ufms.com

Fernando Roberto Ferreira Valente

ORCID: https://orcid.org/0000-0002-8188-2986 Universidade Estadual de Campinas, Brasil E-mail: fernandocropp@terra.com.br

Muryllo Eduardo Sales dos Santos

ORCID: https://orcid.org/0000-0001-8517-4154 Universidade Federal do Mato Grosso do Sul, Brasil E-mail: muryllosales@gmail.com

\section{Resumo}

O queratocisto odontogênico (QO) é um lesão cística intra-óssea, sendo um dos cistos mais comuns dos ossos gnáticos responsável por 3\% a $11 \%$ de todos os cistos odontogênicos. Essa lesão apresenta altas taxas de recidiva, de 5 a $62 \%$, e geralmente acontece nos primeiros 05 anos, e pode estar diretamente ligada ao tipo de técnica utilizada no tratamento da lesão. As formas de tratamento para o QO podem variar de acordo com sua apresentação clínica e imaginologica. Considerando as altas taxas de recidiva dessa patologia benigna apenas a enucleação pode não ser suficiente, então outras formas de tratamento adjuvantes são propostas como a crioterapia. Este relato de caso tem como objetivo apresentar o caso de uma paciente de 22 anos, que apresentou um queratocisto odontogênico (QO) em mandíbula do lado direito com diagnóstico em 2012. Foi realizado o tratamento cirúrgico de enucleação e tratamento adjuvante com crioterapia utilizando gás refrigerante. A paciente segue em acompanhamento por 10 anos, sem queixas e não apresentou nenhuma recidiva do cisto.

Palavras-chave: Cistos ósseos; Crioterapia; Tratamento conservador.

\begin{abstract}
Odontogenic keratocyst $(\mathrm{OKC})$ is an intraosseous cystic lesion, being one of the most common cysts of the gnathic bones, responsible for $3 \%$ to $11 \%$ of all odontogenic cysts. This cyst has high recurrence rates, from 5 to $62 \%$, and usually occurs within the first 5 years, and may be directly linked to the type of technique used to treat the lesion. The forms of treatment for OKC may vary according to its clinical and imaging presentation. Considering the high recurrence rates of this benign pathology, only enucleation may not be enough, so other forms of adjuvant treatment are proposed, such as cryotherapy. This case report aims to present the case of a 22 -year-old female patient who presented an odontogenic keratocyst (OKC) in the right mandible diagnosed in 2012. Surgical treatment of enucleation and adjuvant treatment with cryotherapy using refrigerant gas were performed. The patient has been followed up for 10 years, without complaints and has not had any recurrence of the cyst.
\end{abstract}

Keywords: Bone cysts; Cryotherapy; Conservative treatment.

\section{Resumen}

El queratoquiste odontogénico (QO) es una lesión quística intraósea, siendo uno de los quistes más comunes de los huesos gnáticos, responsable del $3 \%$ al $11 \%$ de todos los quistes odontogénicos. Esta lesión tiene altas tasas de 
recurrencia, del 5 al 62\%, y generalmente ocurre dentro de los primeros 5 años, y puede estar directamente relacionada con el tipo de técnica utilizada para tratar la lesión. Las formas de tratamiento del QO pueden variar según su presentación clínica e imagenológica. Teniendo en cuenta las altas tasas de recurrencia de esta patología benigna, la enucleación sola puede no ser suficiente, por lo que se proponen otras formas de tratamiento adyuvante, como la crioterapia. Este reporte de caso tiene como objetivo presentar el caso de una paciente de sexo femenino de 22 años que presentó un queratoquiste odontogénico (QO) en la mandíbula derecha diagnosticado en el año 2012. Se realizó tratamiento quirúrgico de enucleación y tratamiento adyuvante con crioterapia con gas refrigerante. El paciente ha sido seguido durante 10 años, sin quejas y no ha tenido ninguna recurrencia del quiste.

Palabras clave: Quistes óseos; Crioterapia; Tratamiento conservador.

\section{Introdução}

Previamente já classificado como tumor odontogênico queratocístico (TOQ) devido ao comportamento clínico agressivo e taxas de recidivas importantes, a partir de 2017, pela OMS, essa lesão voltou a ser definida como cisto odontogênico de desenvolvimento. Levou-se em consideração suas características histológicas, além de sua resposta à marsupialização, o que um processo neoplásico não teria. (Barnes et al, 2005; Al Moraissi et al, 2017; Ribeiro-Junior et al., 2017).

O queratocisto odontogênico (QO) é um lesão cística intra-óssea, sendo um dos cistos odontogênicos mais comuns dos ossos gnáticos (Kaczmarzyk et al., 2012; Pitak-Arnnop et al., 2010), responsável por 3\% a 11\% de todos os cistos odontogênicos (Neville et al., 2009). Essa patologia geralmente se apresenta sem sintomas e é encontrada em exames de imagem de rotina, apresentando expansão apenas em estágios mais avançados. O queratocisto inicialmente se estende no sentido anteroposterior sem demonstrar abaulamento das tabuas ósseas. Nas lesões extensas os pacientes podem apresentar dor, movimentações e reabsorções dentárias, aumento volumétrico em face e até fraturas patológicas (Gupta et al., 2016; Díaz-Belenguer et al., 2016).

O QO afeta mais comumente homens, em sua terceira década de vida, e apresenta predileção de mais de $70 \%$ pela mandíbula (Shear et al., 2008), principalmente nas regiões de terceiro molar, angulo, região posterior do corpo e ramo ascendente (Osterne et al., 2011; Karaca et al., 2018; Díaz-Belenguer et al., 2016). Esses cistos exigem atenção especial considerando suas altas taxas de recidiva (Sigua-Rodriguez et al., 2019), que podem variar de 5 a $62 \%$ (Neville et al., 2009). A mesma acontece geralmente nos primeiros 05 anos (Kinard et al., 2013), e pode estar diretamente ligada ao tipo de técnica utilizada no tratamento da lesão (Oliveira et al., 2021).

Ao exame de imagem, geralmente esta associado à terceiros molares não erupcionados, podendo se apresentar uni ou multilocular com bordas escleróticas bem definidas (Chrcanovic et al., 2017; Castro et al., 2018). Em alguns casos, pode-se também observar reabsorção de raízes dentárias ou extrusões dos dentes envolvidos (Gaitán-Cepada et al., 2010). A partir dessas características o diagnóstico diferencial para essa patologia pode ser o cisto dentígero, o cisto periodontal lateral ou o cisto radicular (Neville et al., 2009; Sharif et al, 2015).

As formas de tratamento para o QO podem variar de acordo com sua apresentação clínica e imaginologica. Uma forma de tratamento usual para esse cisto é a enucleação da lesão. Entretanto, em lesões com extensões maiores a marsupialização ou descompressão prévias são utilizadas (Sharif et al, 2015) no intuito de facilitar a enucleação e limitar os riscos de fratura patológica ou para preservar alguma estrutura anatômica importante envolvida na lesão inicialmente.

Considerando as altas taxas de recidiva dessa patologia benigna apenas a enucleação pode não ser suficiente, então outras formas de tratamento adjuvantes foram propostas como a crioterapia (Schmidt, 2003; Santos et al., 2021) ou utilização de agentes esclerosantes, como a solução de Carnoy (Stoelinga, 2005). Terapêuticas mais agressivas também são descritas como a enucleação associada a osteotomia periférica e a ressecção em bloco (Ghali et al., 2003; Oliveira et al., 2021).

O objetivo deste trabalho é descrever o caso clínico de um queratocísto odontogênico em região posterior de mandíbula com envolvimento de terceiro molar incluso, com tratamento de enucleação associada à crioterapia com gás refrigerante propano-butano e acompanhamento de 09 anos sem recidivas. 


\section{Metodologia e Relato de Caso}

O presente relato de caso clínico tem caráter descritivo, exploratório e de abordagem qualitativa como descrito pela literatura (Pereira et al., 2018). A paciente a ser apresentada assinou, um termo de consentimento para divulgação de suas imagens e dados, além das etapas relacionadas ao diagnóstico e todo o seu tratamento no Hospital Universitário Maria Aparecida Pedrossian da Universidade Federal de Mato Grosso do Sul. Ainda assim, é lícito afirmar que foram respeitados todos os princípios éticos da Declaração de Helsinque e independente da assinatura da paciente, o melhor tratamento possível foi realizado.

Paciente de 22 anos de idade, A. G. S., leucoderma, sexo feminino, estudante universitária, procurou atendimento no ambulatório do Serviço de Cirurgia e Traumatologia Buco-maxilo-facial do Hospital Universitário Maria Aparecida Pedrossian da Universidade Federal de Mato Grosso do Sul (UFMS) em janeiro de 2012, tendo sido encaminhada de outro serviço para uma segunda opinião com relação ao tratamento de lesão mandibular. Na ocasião, a paciente trouxe exames de imagem e resultado de biopsia incisional previamente realizada com o diagnóstico de tumor odontogênico queratocistico.

No exame clínico extra oral observou-se discreta assimetria facial do lado direito, e no intraoral, mucosa integra, sem alterações de cor ou ulcerações e ausência de elemento dentário 48. À palpação digital da região, havia discreto abaulamento da tábua óssea vestibular, indolor (Figura 1).

Figura 1. A. Aspecto clínico extra oral. discreta assimetria facial do lado direito. B. Aspecto clínico intraoral, mucosa integra sem ulcerações ou alterações de cor.
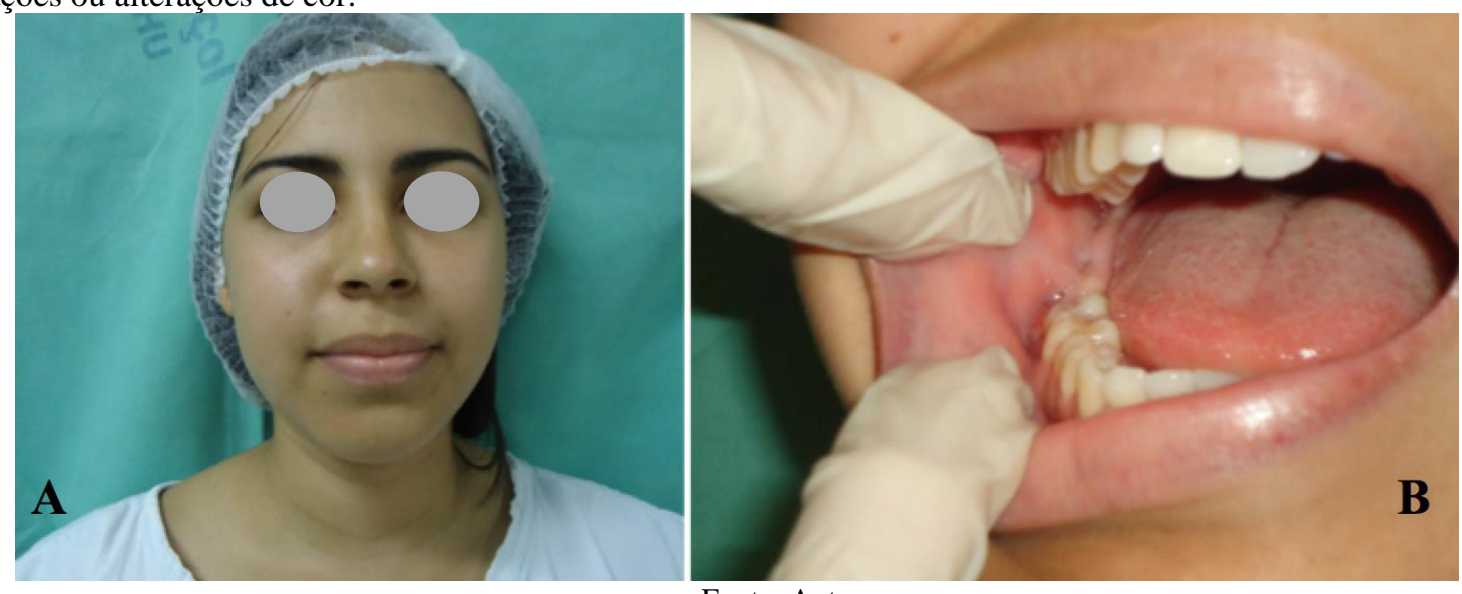

Fonte: Autores.

Os exames de imagem revelaram extensa lesão em corpo e ramo ascendente de mandíbula do lado direito, envolvendo os dentes 46, 47 e o 48 incluso e com deslocamento para região do ramo. Revelaram ainda abaulamento das tabuas ósseas vestibular e lingual, assim como, desvio do feixe vásculo-nervoso para a região basilar. (Figura 2) 
Figura 2. A. Radiografia panorâmica, onde se observa extensa lesão radiolúcida envolvendo os dentes 46, 47 e o 48 deslocado para o ramo mandibular. Observa-se ainda envolvimento da região basilar. B. Tomografia computadorizada (TC) - corte axial, onde se observa extensa lesão hipodensa e abaulamento das tabuas ósseas vestibular e lingual. C. TC 3D onde se observa fenestração das tabuas ósseas vestibular e lingual e deslocamento do feixe vásculo-nervoso para região basilar.

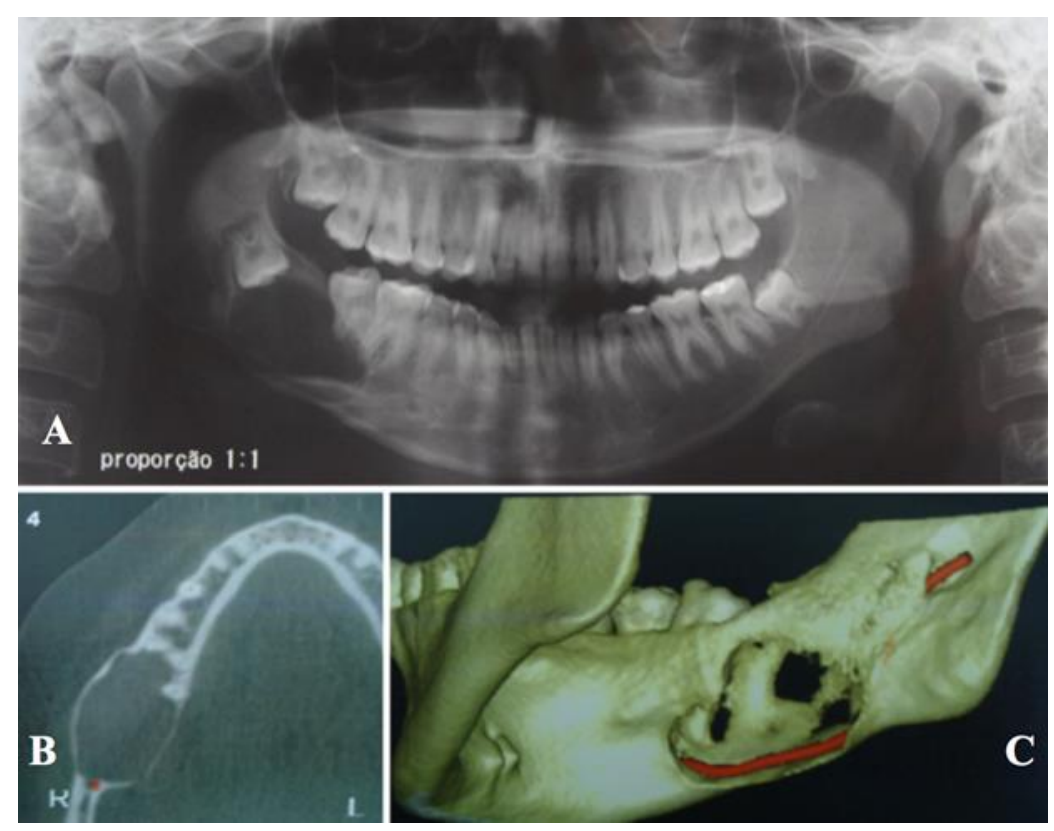

Fonte: Autores.

Diante do quadro, se propôs realizar o tratamento da lesão. O tratamento proposto foi realizar a enucleação da lesão com crioterapia adjuvante utilizando-se gás refrigerante (Endo-Frost®, Roeko, Langenau, Alemanha).

O procedimento foi realizado em centro cirúrgico, sob anestesia geral, com intubação nasoendotraqueal. Realizou-se antissepsia extraoral com clorexidina $0,5 \%$ e intraoral à $0,12 \%$, seguida de isolamento do campo operatório. Realizou-se infiltração anestésica na região de corpo e ramo mandibular do lado direito, intraoralmente, com lidocaína $2 \%$ com adrenalina 1:100.000, com fins de se obter melhor hemostasia.

Realizou-se uma incisão intrasulcular, com lamina de bisturi n.15, na região dos elementos dentários 46 e 47 e linear na região retromolar. Seguiu-se com o descolamento mucoperiosteal e a exposição da área de interesse. Os elementos dentários 47 e 48 envolvidos na lesão foram removidos. Seguiu-se com a enucleação e curetagem da lesão e tratamento da loja cirúrgica, após o quê, realizou-se a crioterapia com gás refrigerante propano-butano (Endo-Frost@, Roeko, Langenau, Alemanha) (Figura $3)$. 
Figura 3. A. Incisão intrasulcular em região de 46, 47 e região retromolar e exposição da área afetada. B. Aplicação do gás refrigerante propano-butano na região.

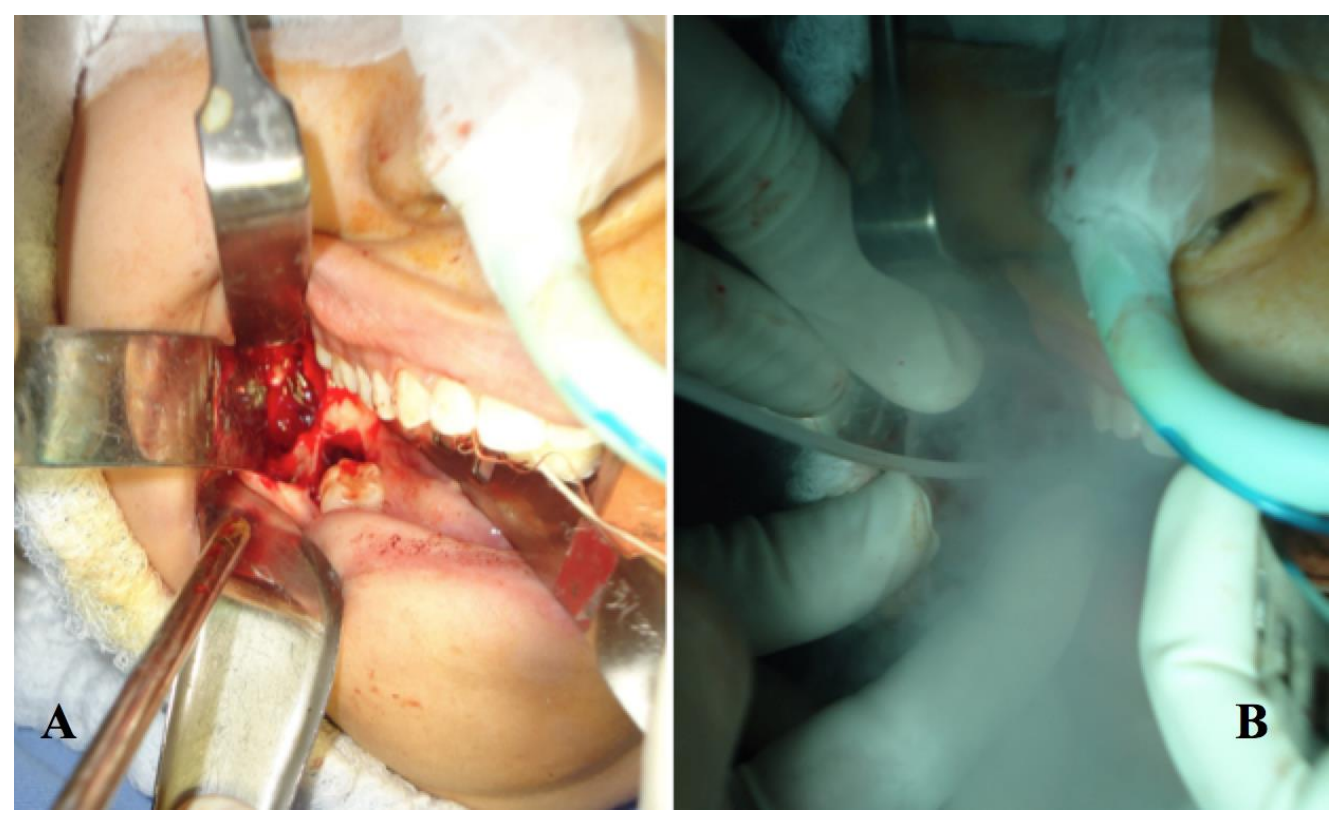

Fonte: Autores.

A crioterapia foi aplicada da seguinte maneira: 3 aplicações de 10 segundos cada, com intervalos de 5 minutos entre as aplicações. A região do feixe vásculo-nervoso foi previamente protegida com gaze, para diminuir os danos ao mesmo. Seguiuse com a irrigação da loja cirúrgica com soro fisiológico $0,9 \%$, e fechamento da ferida com fio nylon n. 5.0.

O material obtido foi encaminhado para análise anatomohistopatológica, e o diagnóstico obtido foi de queratocisto odontogênico, corroborando com o diagnóstico inicial.

A paciente tem sido acompanhada ao longo do tempo, durante 10 anos. Aos 6 meses pós operatório a paciente apresentava-se bem, sem queixas e o exame de imagem mostrou processo cicatricial da região com neoformação óssea adequada. Aos 4 anos e 9 meses, a radiografia panorâmica digital mostrou completa neoformação óssea da região, e com o feixe vásculonervoso em posição anatômica, sem deslocamento (Figura 4). Aos 10 anos, a TC Cone Beam volumétrica numa vista frontal e lateral direita revelaram completa cicatrização do tecido ósseo e sem sinais de recidiva (Figura 5). 
Figura 4. A. Radiografia panorâmica de 6 meses pós operatória, onde se observa neoformação óssea em estágio inicial. B. Radiografia panorâmica digital aos 4 anos e 9 meses, onde se observa completa neoformação óssea e o feixe vásculo-nervoso sem deslocamento e em sua posição anatômica.

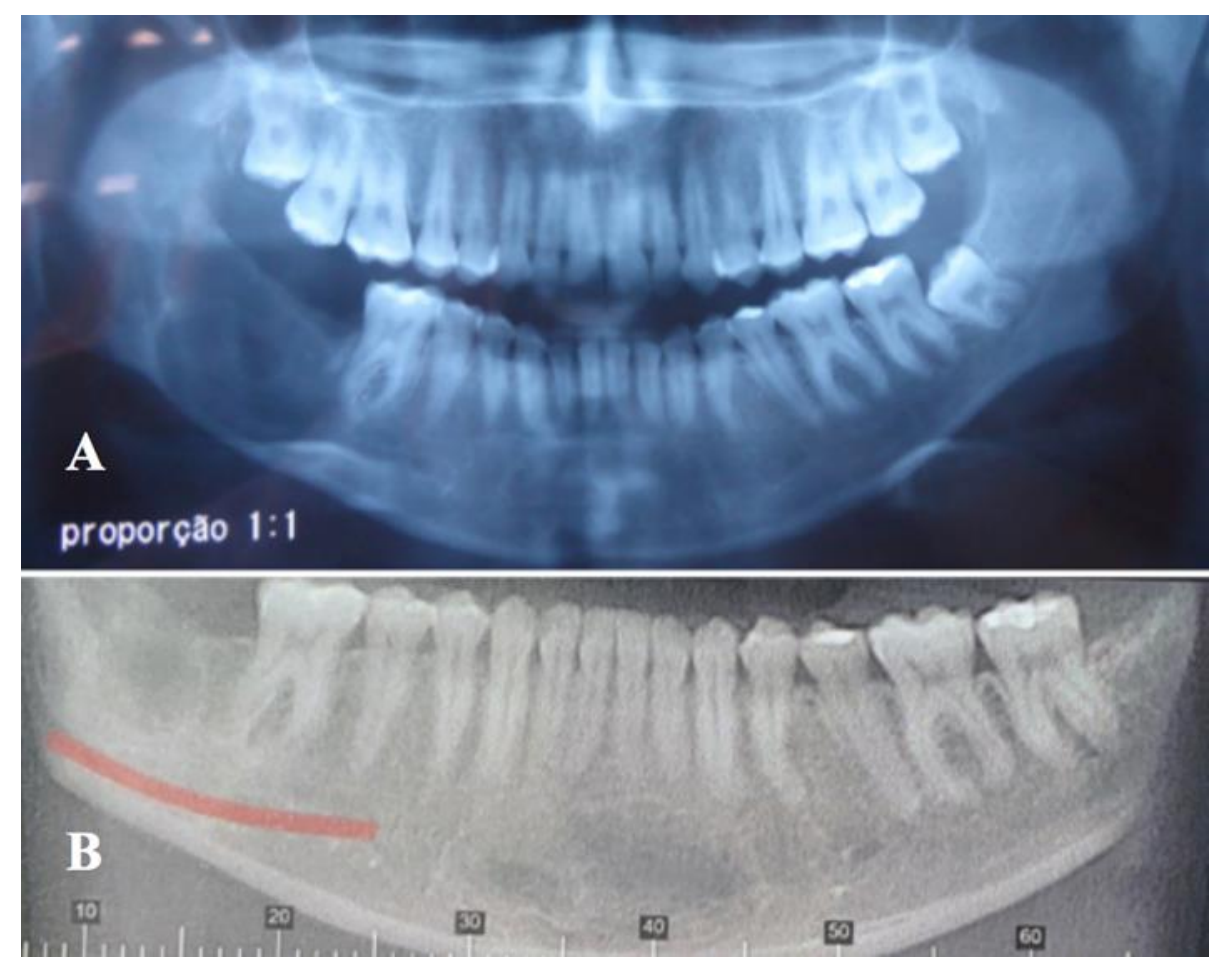

Fonte: Autores.

Figura 5. A. TC corte axial, onde se observa completa cicatrização do tecido ósseo, sem expansão das corticais. B. TC Cone Beam volumétrica, vista lateral, onde se observa volume ósseo adequado, sem sinais de recidiva.
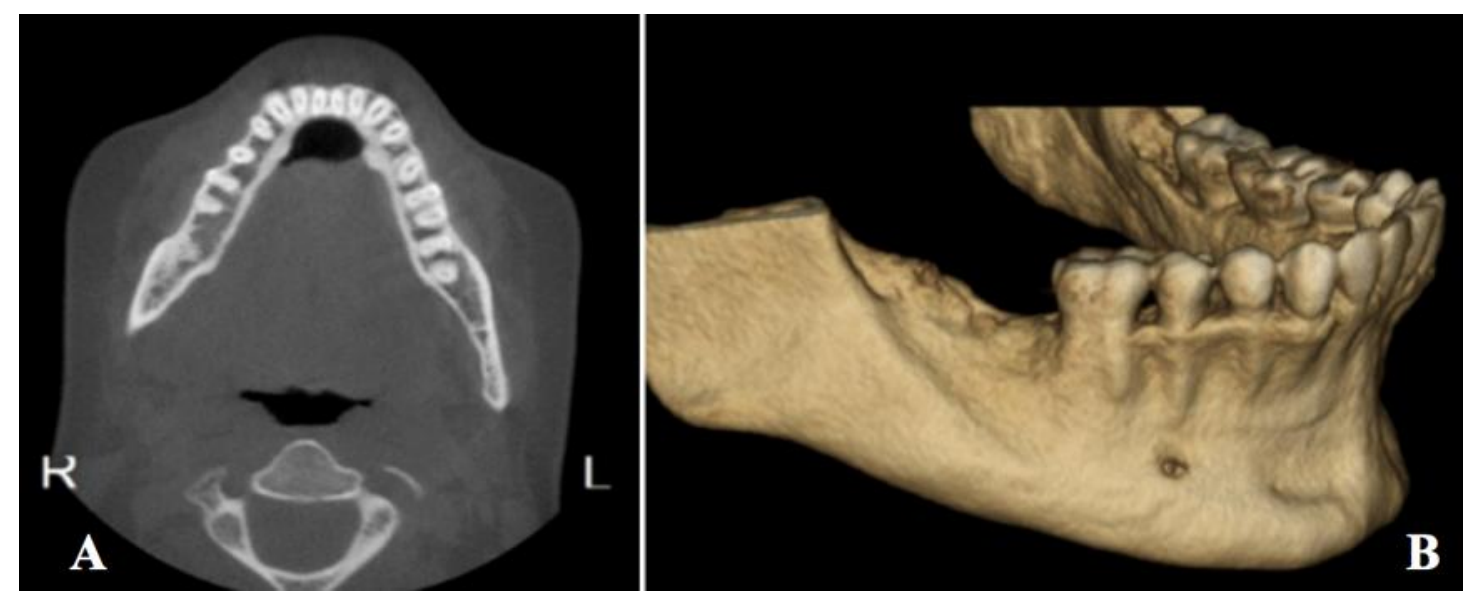

Fonte: Autores.

\section{Discussão}

São diversas as opções de tratamento para o queratocisto odontogênico (Morgan et al., 2005; Kaczmarzyk et al., 2012, Oliveira et al., 2021). Variando entre as mais conservadoras, como a marsupialização e enucleação simples, às mais agressivas como a ressecção em bloco. Entretanto, ainda não existe consenso na literatura quanto ao melhor manejo para esse tipo de lesão, principalmente considerando as altas taxas de recidiva variando de 5\% a 62\% (Neville et al., 2009).

É certo que dentre os tratamentos mais conservadores as taxas de recidivas são maiores. Considerando isso, alguns 
tratamentos adjuvantes são opções importantes no tratamento deste cisto, como a osteotomia periférica, uso da solução de Carnoy ou crioterapia após a enucleação da lesão (Fonseca et al., 2010). É controverso qual método adjuvante pode oferecer menor possibilidade que o cisto retorne (Morgan et al., 2005; Kaczmarzyk et al., 2012, Oliveira et al., 2021).

Neste relato de caso, utilizou-se a técnica de enucleação do QO e crioterapia adjuvante. A crioterapia utilizada foi realizada com um gás refrigerante propano-butano. Apesar de ser mais comum a utilização de nitrogênio líquido na crioterapia adjuvante, o gás refrigerante propano-butano tem demonstrado bons resultados, e é de baixo custo e fácil aquisição (Mendonça et al., 2011; Benaglia et. al., 2014; Benaglia, 2014).

Segundo Moura-Netto et al., 2007 o Endo-Frost (Endo-Frost®, Roeko, Langenau, Alemanha) atinge temperatura de 50oC. Como reação local, há uma lesão celular direta resultante da crionecrose a partir da exposição à temperatura negativa extrema (Barbosa \& Sanvitto, 1973; Costa et al., 2010; Bansal et al., 2012; Benaglia, 2014). Deste modo, como corroborado pela literatura, este spray mostra viabilidade terapêutica, onde sabe-se que para que ocorra a morte celular devem ser utilizadas temperaturas abaixo de -20 oC (Leopard, 1975; Tal, 1982; Gage et al., 2009).

Outros estudos, para avaliação desse gás refrigerante para tratamento de lesões orais, como o descrito por Benaglia, 2014 demonstram bons resultados em ratos. Os autores descrevem que o uso do gás refrigerante propano-butano apresentou o mesmo resultado obtido com o nitrogênio líquido, obtendo a crionecrose, além de apresentar melhor tempo de cicatrização nos espécimes observados. Além disso, outros casos clínicos já descritos também demonstram resultados satisfatórios dessa técnica adjuvante utilizando o spray (Mendonça et. al., 2011, Santos et al., 2021).

Nos procedimentos criocirúrgicos utilizando o nitrogênio líquido, pode-se obter temperatura potencial de $-196^{\circ} \mathrm{C}$, sendo amplamente utilizada como padrão ouro no tratamento de lesões da cavidade oral. Entretanto, exige-se aparelhagem específica, com contêiner próprio, criocautério com válvula e ponteiras (Bansal et al., 2012). Entretanto, a crioterapia com o gás refrigerante Endo-Frost, é simples, não exige aparelhagem sofisticada e apresenta menor custo. E neste caso foi realizada em três sessões de 10 segundos, com intervalos de 5 minutos entre as sessões de acordo com o preconizado por Benaglia, 2014.

O tratamento deste tipo de lesão, de acordo com a literatura, poderia ser realizado com a ressecção em bloco, o que poderia causar deformidade facial de difícil resolução ou reabilitação (Castro et al., 2018; Slusarenko da Silva, Stoelinga et al., 2019; Slusarenko da Silva et al., 2019). Tratando-se de uma paciente jovem e que não aceitava a possibilidade de tratamento radical, optou-se pelo tratamento mais conservador, mesmo sabendo-se do risco potencial de recidiva e/ou fratura patológica (Sigua-Rodriguez et al., 2019; Oliveira et al., 2021)

A paciente esta sendo monitorada até a presente data, sem queixas, satisfeita com o resultado e os exames de imagem após 10 anos não mostram sinais de recidiva.

\section{Conclusão}

Não existe na literatura um consenso quanto ao melhor tratamento para o queratocisto. No entanto, a maioria dos autores preconizam um tratamento mais radical com remoção em bloco.

A enucleação seguida da crioterapia adjuvante mostrou ser eficaz num período de 10 anos, neste relato de caso. A técnica utilizada evitou que a paciente sofresse mutilações de difícil resolução.

A crioterapia com gás refrigerante é de baixo custo, sendo um produto multiuso e de fácil acesso ao cirurgião dentista. Entretanto, novos estudos são necessários para aperfeiçoamento da técnica no tratamento das lesões da cavidade oral.

\section{Agradecimentos}

O presente trabalho foi realizado com apoio da Fundação Universidade Federal de Mato Grosso do Sul -UFMS/MEC - Brasil. 


\section{Referências}

Al-Moraissi, E. A., Dahan, A. A., Alwadeai, M. S., Oginni, F. O., Al-Jamali, J. M., Alkhutari, A. S., \& Al-Sanabani, J. S. (2017). What surgical treatment has the lowest recurrence rate following the management of keratocystic odontogenic tumor?: A large systematic review and meta-analysis. Journal of CranioMaxillofacial Surgery. 45(1), 131-144.

Bansal, A., Jain, S., \& Gupta, S. (2012). Cryosurgery in the treatment of oro-facial lesions. Indian Journal of Dental Research. 23(2), 297-302.

Barbosa, J. F., \& Sanvitto, L. C. (1973). Crioterapia local (Criocirurgia)-denominação e histórico. Boletim de Oncologia. 3(3-4), $29-34$.

Barnes, L., Eveson, J. W., Sidransky, D., \& Reichart, P. (Eds.). (2005). Pathology and genetics of head and neck tumours. Vol. 9. IARC Press.

Benaglia, M. B. (2014). Estudo morfológico comparativo da crioterapia com nitrogênio líquido e gás refrigerante propano-butano na mucosa bucal de ratos. (dissertação de mestrado, Universidade Federal de Mato Grosso do Sul, Campo Grande, MS). https://repositorio.ufms.br/browse?type=subject\&value=Nitrogen

Benaglia, M. B., Jardim, E. C. G., \& Mendonca, J. C. (2014). Criocirurgia em odontologia: vantagens e desvantagens. Brazilian Journal of Surgery and Clinical Research. 7(3), 58-67.

Castro, M. S., Caixeta, C. A., de Carli, M. L., Ribeiro Junior, N. V., Miyazawa, M., Pereira, A. A. C., ... \& Hanemann, J. A. C. (2018). Conservative surgical treatments for nonsyndromic odontogenic keratocysts: a systematic review and meta-analysis. Clinical Oral Investigations. 22(5), $2089-2101$.

Chrcanovic, B. R., \& Gomez, R. S. (2017). Recurrence probability for keratocystic odontogenic tumors: an analysis of 6427 cases. Journal of cranioMaxillofacial Surgery. 45(2), 244-251.

Costa, F. W. G., Soares, E. C. S., \& Batista, S. H. B. (2010). Criocirurgia no tratamento de lesões benignas dos maxilares: revisão de literatura e análise de 103 casos previamente reportados. RSBO Revista Sul-Brasileira de Odontologia. 7(2), 208-215.

Slusarenko da Silva, Y., Stoelinga, P. J., \& Naclério-Homem, M. D. G. (2019). Recurrence of nonsyndromic odontogenic keratocyst after marsupialization and delayed enucleation vs. enucleation alone: a systematic review and meta-analysis. Oral and Maxillofacial Surgery. 23(1), 1-11.

Slusarenko da Silva, Y., Stoelinga, P. J., \& Naclério-Homem, M. D. G. (2019). The presentation of odontogenic keratocysts in the jaws with an emphasis on the tooth-bearing area: a systematic review and meta-analysis. Oral and Maxillofacial Surgery. 23(2), 133-147.

Oliveira, M. H., de Oliveira Aguillera, M., dos Santos, M. E. S., Figueiredo, F. T., Nejaim, Y., Antunes, D. M., \& Jardim, E. C. G. (2021). Queratocisto odontogênico: índice de recorrência em função do tipo de tratamento. Archives Of Health Investigation. 10(9), 1396-1402.

Díaz-Belenguer, Á., Sánchez-Torres, A., \& Gay-Escoda, C. (2016). Role of Carnoy’s solution in the treatment of keratocystic odontogenic tumor: a systematic review. Medicina Oral, Patologia Oral Y Cirugia Bucal. 21(6), 689-695.

Santos, A. O. G. M., de Mendonça, J. C. G., Pelissaro, G. S., Paiva-Oliveira, J. G., da Silva, J. C. L., de Oliveira Aguillera, M., \& Jardim, E. C. G. (2021). Curetagem cirúrgica associada à crioterapia como alternativa para tratamento de mixoma odontogênico em maxila: Relato de um caso clínico. Research, Society and Development. 10(10), e103101018693-e103101018693.

Fonseca, E. V., Franzi, S. A., Marcucci, M., \& De Almeida, R. C. (2010) Clinical, histopathological and treatment factor of the odontogenic keratocyst. Brazilian Journal of Head and Neck Surgery. 39(1), 57-61.

Gage, A. A., Baust, J. M., \& Baust, J. G. (2009). Experimental cryosurgery investigations in vivo. Cryobiology. 59(3), $229-243$.

Gaitán-Cepeda, L. A., Quezada-Rivera, D., Tenorio-Rocha, F., \& Leyva-Huerta, E. R. (2010). Reclassification of odontogenic keratocyst as tumour. Impact on the odontogenic tumours prevalence. Oral Diseases. 16(2), 185-187.

Ghali, G. E., \& Connor, M. S. (2003). Surgical management of the odontogenic keratocyst. Oral and Maxillofacial Surgery Clinics. 15(3), 383-392.

Gupta, A., Bansal, P., Sharma, R., \& Sharma, S. D. (2016). Treatment of keratocystic odontogenic tumours: a prospective study of 30 cases. Journal of Maxillofacial and Oral Surgery, 15(4), 521-527.

Kaczmarzyk, T., Mojsa, I., \& Stypulkowska, J. (2012). A systematic review of the recurrence rate for keratocystic odontogenic tumour in relation to treatment modalities. International Journal of Oral and Maxillofacial Surgery. 41(6), 756-767.

Karaca, Ç., Dere, K. A., Er, N., Aktaş, A., Tosun, E., Köseoğlu, O. T., \& Usubütün, A. (2018). Recurrence rate of odontogenic keratocyst treated by enucleation and peripheral ostectomy: Retrospective case series with up to 12 years of follow-up. Medicina Oral, Patología Oral Y Cirugía Bucal. $23(4)$, e443.

Kinard, B. E., Chuang, S. K., August, M., \& Dodson, T. B. (2013). How well do we manage the odontogenic keratocyst?. Journal of Oral and Maxillofacial Surgery. 71(8), 1353-1358.

Leopard, P. J. (1975). Cryosurgery, and its application to oral surgery. The British Journal of Oral Surgery. 13(2), 128-152.

Mendonça, J. C. G., Santos, A. A., \& Lopes, H. B. (2011). Hemimaxilectomia associada à crioterapia no tratamento de ameloblastoma: relato de caso. Revista Brasileira de Cirurgia Craniomaxilofacial. 14(1), 63-6.

Morgan, T. A., Burton, C. C., \& Qian, F. (2005). A retrospective review of treatment of the odontogenic keratocyst. Journal of Oral and Maxillofacial Surgery. $63(5), 635-639$

Moura-Netto, C., Yamazaki, A. K., Cardoso, L. N., Cabrales, R. J. S., \& Prokopowitsch, I. (2007). Avaliação da temperatura mínima alcançada por cinco gases refrigerantes Lowest temperature reached by five cold spray gases. Revista do Instituto de Ciências da Saúde. 25(4), 403-5. 
Neville, B. W., Damm, D. D., Allen, C. M., \& Bouquot, J. E. (2009) Patologia Oral e Maxilofacial. Elsevier.

Osterne, R. L. V., de Matos Brito, R. G., Alves, A. P. N. N., Cavalcante, R. B., \& Sousa, F. B. (2011). Odontogenic tumors: a 5-year retrospective study in a Brazilian population and analysis of 3406 cases reported in the literature. Oral Surgery, Oral Medicine, Oral Pathology, Oral Radiology, and Endodontology. 111(4), 474-481.

Pitak-Arnnop, P., Chaine, A., Oprean, N., Dhanuthai, K., Bertrand, J. C., \& Bertolus, C. (2010). Management of odontogenic keratocysts of the jaws: a ten-year experience with 120 consecutive lesions. Journal of Cranio-Maxillofacial Surgery. 38(5), 358-364.

Ribeiro-Júnior, O., Borba, A. M., Alves, C. A. F., Gouveia, M. M. D., Deboni, M. C. Z., \& Naclério-Homem, M. D. G. (2017). Reclassification and treatment of odontogenic keratocysts: A cohort study. Brazilian Oral Research. 31.

Schmidt, B. L. (2003). The use of liquid nitrogen cryotherapy in the management of the odontogenic keratocyst. Oral and Maxillofacial Surgery Clinics. 15(3), $393-405$.

Sharif, F. N., Oliver, R., Sweet, C., \& Sharif, M. O. (2015). Interventions for the treatment of keratocystic odontogenic tumours. Cochrane database of systematic reviews. 11.

Shear, M., \& Speight, P. (2008). Cysts of the oral and maxillofacial regions. John Wiley \& Sons.

Sigua-Rodriguez, E. A., Goulart, D. R., Sverzut, A., Asprino, L., \& de Moraes, M. (2019). Is surgical treatment based on a 1-step or 2-step protocol effective in managing the odontogenic keratocyst?. Journal of Oral and Maxillofacial Surgery. 77(6), 1210-e1.

Stoelinga, P. J. (2005). The treatment of odontogenic keratocysts by excision of the overlying, attached mucosa, enucleation, and treatment of the bony defect with carnoy solution. Journal of Oral and Maxillofacial Surgery. 63(11), 1662-1666.

Tal, H. (1982). The rationale and technique of cryotherapy of superficial oral lesions. The Journal of the Dental Association of South Africa. 37(5), 335-340. 